\title{
Improvement and Interlaboratory Validation of the Lipophilic Oxygen Radical Absorbance Capacity: Determination of Antioxidant Capacities of Lipophilic Antioxidant Solutions and Food Extracts
}

\author{
Jun Watanabe, ${ }^{* \dagger}$ Tomoyuki OKI, ${ }^{* *}$ Jun TAKEBAYAShI, ${ }^{* * *}$ Hiroshi YADA,* Manabu WAGaKI,* \\ Yuko TAKANO-ISHIKAWA,* and Akemi YASUI* \\ *National Food Research Institute, National Agriculture and Food Research Organization, \\ 2-1-12 Kannondai, Tsukuba 305-8642, Japan \\ **National Agricultural Research Center for Kyushu Okinawa Region, National Agriculture and Food \\ Research Organization, 2421 Suya, Koshi, Kumamoto 861-1192, Japan \\ ***National Institutes of Biomedical Innovation, Health and Nutrition, National Institute of Health and \\ Nutrition, 1-23-1 Toyama, Shinjuku, Tokyo 162-8636, Japan
}

\begin{abstract}
A lipophilic oxygen radical absorbance capacity (L-ORAC) assay is an evaluation of the antioxidant capacity of solutions of lipophilic compounds. The concentrations of fluorescein, radical generator, and Trolox standard solutions were optimized to improve the precision of the assay. An interlaboratory study using two antioxidant solutions and three food extracts as test samples conducted in accordance with harmonized protocol demonstrated satisfactory L-ORAC measurements; the intermediate precision relative standard deviations $\left(\mathrm{RSD}_{\text {int }}\right)$ ranged from 7.0 to $16.7 \%$, the reproducibility relative standard deviations $\left(\mathrm{RSD}_{\mathrm{R}}\right)$ ranged from 14.8 to $19.4 \%$, and the HorRat values ranged from 1.35 to 1.78.
\end{abstract}

Keywords Lipophilic oxygen radical absorbance capacity, antioxidant capacity, method validation

(Received July 7, 2015; Accepted August 24, 2015; Published February 10, 2016)

\section{Introduction}

Dietary antioxidants are believed to play a crucial role in human health by protecting biological molecules from oxidative damage in the body. It has been reported that total dietary antioxidant capacity is inversely associated with the risks of stroke $^{1}$ and cardiovascular disease. ${ }^{2}$

The oxygen radical absorbance capacity (ORAC) assay measures the scavenging capacity against peroxyl radicals induced by 2,2'-azobis(2-amidinopropane) dihydrochloride (AAPH) using fluorescein as a fluorescence probe. ${ }^{3}$ The assay is typically conducted in a 96-well microplate. The antioxidant capacity of a sample is evaluated by assessing the area under the fluorescence decay curve using a microplate reader. 6-Hydroxy2,5,7,8-tetramethylchroman-2-carboxylic acid (Trolox) is used as a standard antioxidant, and the antioxidant capacity of a sample is expressed as Trolox equivalent (TE). The ORAC assay has been widely used because of its advantages related to biological systems. ${ }^{4}$

Fruits and vegetables contain both hydrophilic antioxidants, such as vitamin $\mathrm{C}$ and the majority of polyphenolic compounds, and lipophilic antioxidants, such as vitamin E and carotenoids. 5 Although the ORAC assay was initially developed to evaluate

$\dagger$ To whom correspondence should be addressed.

E-mail: nabej@affrc.go.jp the activity of hydrophilic antioxidants, ${ }^{3}$ Huang et al. ${ }^{6}$ extended the ORAC assay to lipophilic antioxidants using randomly methylated $\beta$-cyclodextrin (RMCD) as a solubility enhancer, thereby allowing measurement of the antioxidant capacities of both lipophilic and hydrophilic components in a given sample using the same peroxyl radical source. Cyclodextrins are cyclic (R-1,4)-linked oligosaccharides of R-D-glucopyranose containing a relatively hydrophobic central cavity and hydrophilic outer surface. This property of cyclodextrin has made it increasingly popular as a vehicle for enhancing the solubility of lipophilic compounds in an aqueous environment in pharmaceutical and food industries. ${ }^{7}$

Wu et al. ${ }^{8}$ reported that the ORAC values of the hydrophilic fractions (H-ORAC) of fruits and vegetables were much higher than those of the lipophilic fractions (L-ORAC). However, this does not mean that the L-ORAC values of fruits and vegetables are negligible. Unlike hydrophilic antioxidants, which do not accumulate in the body and are excreted in the urine, lipophilic antioxidants penetrate the lipoprotein cell membrane more easily and therefore attain a higher level of bioavailability. ${ }^{9}$ For example, the L-ORAC values, but not the H-ORAC values, of broccoli extracts correlated with the prevention of reactive oxygen compound-induced oxidative stress in HepG2 human hepatoma cells, suggesting the high bioavailability of lipophilic antioxidants in vegetables. ${ }^{10}$

We previously reported an improved method for H-ORAC determination that demonstrates satisfactory performance in 
Table 1 Procedural differences between methods A and B

\begin{tabular}{lll}
\hline & & Method A \\
\hline Concentration of fluorescein & $110.7 \mathrm{nmol} / \mathrm{L}$ & $77.5 \mathrm{nmol} / \mathrm{L}$ \\
Concentration of AAPH & $63.4 \mathrm{mmol} / \mathrm{L}$ & $82.4 \mathrm{mmol} / \mathrm{L}$ \\
Concentration of Trolox standard solutions & $100,75,50,25 \mu \mathrm{mol} / \mathrm{L}$ & $160,80,40,20 \mu \mathrm{mol} / \mathrm{L}$ \\
Dilution ratios for the second step measurement & Lower: Approximate L-ORAC divided by 60 Lower: Approximate L-ORAC divided by 80 \\
& Higher: 1.5 times of lower dilution ratio & Higher: 2-fold of lower dilution ratio \\
Positive and negative controls & No & Yes
\end{tabular}

interlaboratory tests using antioxidant solutions and food extracts. ${ }^{11}$ In an earlier study of L-ORAC assays, ${ }^{12}$ we revealed that the repeatability and intermediate precision of the original L-ORAC method ${ }^{13}$ are inadequate, and thus we developed a modified method that provides satisfactory repeatability and intermediate precision in a single-laboratory validation study. Here, we conducted interlaboratory tests in accordance with an internationally harmonized protocol for the design of method-performance studies ${ }^{14}$ to validate this improved L-ORAC method.

\section{Experimental}

\section{Reagents and chemicals}

RMCD was purchased from Junsei Chemical Co., Ltd. (Tokyo, Japan). Fluorescein and Trolox were purchased from Aldrich (Milwaukee, WI). AAPH was obtained from Wako Pure Chemicals Industries (Osaka, Japan). Lipophilic antioxidants ( $\alpha$-tocopherol, $\quad \gamma$-oryzanol, 3,5-di-t-butyl-4-hydroxytoluene (BHT), $t$-butylhydroxyanisole (BHA), and 2,6-di-t-butyl- $p$-cresol (DBPC)) were purchased from Wako Pure Chemicals Industries. All other chemicals were reagent grade.

\section{Test samples}

$\alpha$-Tocopherol, $\gamma$-oryzanol, BHT, BHA, and DBPC were individually dissolved in dimethylsulfoxide (DMSO) at a concentration of $1 \mathrm{~g} / \mathrm{L}$. Dried powders of cinnamon and ginger were purchased from retail stores in Ibaraki, Japan, in July 2014. Unpolished "Koshihikari" rice was pulverized using a grinder mill (GM-200; Retsch, Haan, Germany). Cinnamon and unpolished rice powders (22 $\mathrm{g}$ each) and ginger powder $(11 \mathrm{~g})$ were divided into several batches and were extracted with $n$-hexane-dichloromethane (1:1) using an accelerated solvent extractor (ASE-350; Dionex, San Jose, CA) as described by Wu et al. ${ }^{8}$ The extracts were pooled and dried under a nitrogen gas flow, and then re-dissolved in DMSO $(110 \mathrm{~mL})$. Aliquots (about $1.8 \mathrm{~mL}$ ) of each antioxidant solution or extract were portioned into 60 glass vials; four vials were used for the homogeneity test, and the remainder was stored at $-80^{\circ} \mathrm{C}$ until transport to the participating laboratories. Positive and negative controls for the measurements were $800 \mathrm{mg} / \mathrm{L}$ of Trolox in DMSO, and DMSO without any antioxidant, respectively. Aliquots (about $1.8 \mathrm{~mL}$ ) of each control were portioned into glass vials, and were stored at $-80^{\circ} \mathrm{C}$ until transport to the participating laboratories.

\section{Homogeneity of the test samples}

Four samples (units) of each test material were taken at random from the filling sequence, and each sample was split into two equal parts (unit subsamples). The L-ORAC values of each unit subsample were measured as described below under repeatability conditions. The within- and between-unit standard deviations for the L-ORAC values were calculated by one-way analysis of variance (ANOVA) and by applying the $F$-test at the $95 \%$ confidence level. All $p$ values of the test materials for the validation study were larger than 0.05 , indicating that the samples were homogeneous.

\section{Stability of the test samples}

The stabilities of the antioxidant solutions and food extracts were verified as previously described ${ }^{11}$ prior to the interlaboratory study. The results showed no significant changes occurred during storage at $4{ }^{\circ} \mathrm{C}$ for 4 weeks. Consequently, all of test samples were packed in insulated boxes, along with a cooling bag, and sent to participants in chilled containers. Upon receipt of the test samples, the participants were asked to immediately store the test samples in a freezer $\left(-20^{\circ} \mathrm{C}\right)$ until use. In addition, samples were to be analyzed within three weeks to ensure that they were still stable upon analysis.

\section{L-ORAC measurement by method A}

We reported this method previously. ${ }^{12}$ The L-ORAC values of the test samples were obtained in two steps. Test samples were diluted 10-fold with diluent (7\% (w/v) RMCD in 50\% (v/v) aqueous acetone), and were then further diluted with $10 \%(\mathrm{v} / \mathrm{v})$ DMSO in the diluent. Samples were diluted in a glass vial using a positive-displacement microdispenser (Model 500; Drummond, Broomall, PA, and Acura 841; Socorex, Ecublens, Switzerland) equipped with a glass capillary or using an automated analytical syringe (eVol; SGE Analytical Science, Austin, TX).

\section{L-ORAC measurement by method B}

Method B is an improvement of method A, and the L-ORAC values of the test samples were similarly obtained on two steps. The procedural differences between the two methods are summarized in Table 1. A typical plate layout for the first- and second-step measurements are shown in Figs. 1a and 1b, respectively. The positive and negative control solutions were diluted 10-fold with the diluent, and then further diluted 4- and 8 -fold with $10 \%$ (v/v) DMSO in the diluent; these controls were used to ensure the validity of the measurements. The following conditions had to be fulfilled in order for the L-ORAC values of a laboratory to be accepted: i) the coefficients of variation of the net area under the curve (net AUC) of all Trolox standard solutions were less than $15 \%$; ii) the net AUC of the negative control solution was between 80 to $120 \%$ that of the blank; iii) the estimated L-ORAC value of the positive control sample was between 2400 and $4000 \mu \mathrm{mol} \mathrm{TE} / \mathrm{L}$ ( $75-125 \%$ of the theoretical L-ORAC value).

\section{Interlaboratory studies of L-ORAC measurement}

Interlaboratory studies were performed in accordance with the internationally harmonized protocol ${ }^{14}$ recommended by CAC/GL 28. Thirteen laboratories participated in 
(a)

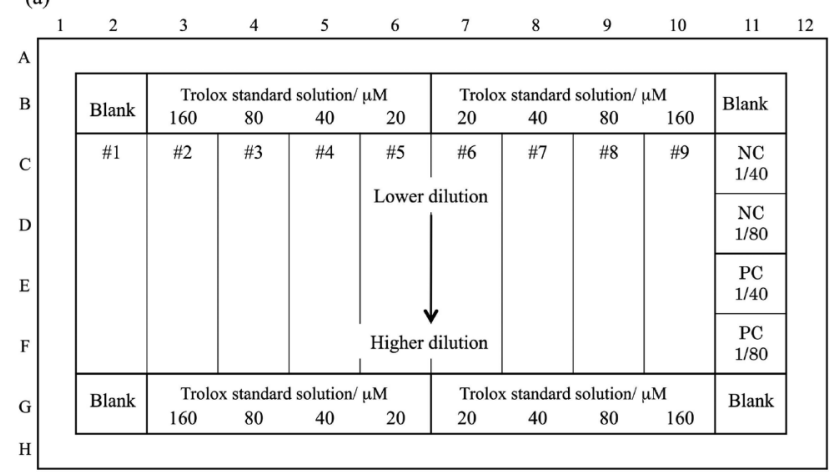

(b)

\begin{tabular}{|c|c|c|c|c|c|c|c|c|c|c|}
\hline & 2 & 3 & 4 & 5 & 6 & 7 & 8 & 9 & 10 & 11 \\
\hline \multicolumn{11}{|l|}{$\mathrm{A}$} \\
\hline \multirow{2}{*}{ B } & & \multicolumn{4}{|c|}{ Trolox standard solution/ $\mu \mathrm{M}$ ) } & \multicolumn{4}{|c|}{ Trolox standard solution $/ \mu \mathrm{M}$} & Blank \\
\hline & Blank & 160 & 80 & 40 & 20 & 20 & 40 & 80 & 160 & Dialik \\
\hline \multirow{2}{*}{$\mathrm{C}$} & \#1 & \#2 & $\# 3$ & $\# 4$ & $\# 5$ & \#6 & $\# 7$ & $\# 8$ & \#9 & $\mathrm{NC}$ \\
\hline & & & & & \multicolumn{2}{|c|}{ Lower dilution } & & & & $1 / 40$ \\
\hline D & & & & & \multicolumn{2}{|c|}{ Higher dilution } & & & & $\begin{array}{l}\mathrm{NC} \\
1 / 80\end{array}$ \\
\hline \multirow{2}{*}{$\mathrm{E}$} & \#9 & \#8 & \#7 & $\# 6$ & $\# 5$ & \#4 & $\# 3$ & \#2 & $\# 1$ & PC \\
\hline & & & & & \multicolumn{2}{|c|}{ Higher dilution } & & & & $1 / 40$ \\
\hline $\mathbf{F}$ & & & & & \multicolumn{2}{|c|}{ Lower dilution } & & & & $\begin{array}{l}\mathrm{PC} \\
1 / 8\end{array}$ \\
\hline \multirow{2}{*}{$\mathrm{G}$} & Blank & \multicolumn{4}{|c|}{ Trolox standard solution/ $\mu \mathrm{M}$} & \multicolumn{4}{|c|}{ Trolox standard solution $/ \mu \mathrm{M}$} & Blank \\
\hline & & 160 & 80 & 40 & 20 & 20 & 40 & 80 & 160 & \\
\hline
\end{tabular}

Fig. 1 Typical plate layout for a preliminary estimate of the L-ORAC value (a), and that for determining the L-ORAC value by method B. The same sample at the same dilution was dispensed into two wells located symmetrically with respect to a point for determining the L-ORAC value (b).

the interlaboratory study of method A and five antioxidant solutions ( $\alpha$-tocopherol, $\gamma$-oryzanol, BHT, BHA, and DBPC) were used whose intra-laboratory precisions had been determined by a single-laboratory test. Sixteen laboratories participated in the interlaboratory study of method B using two antioxidant solutions ( $\alpha$-tocopherol and $\gamma$-oryzanol) and three food extracts. Participants received a shipment containing 10 containers of test samples: two sets of test samples, with each set consisting of five different solutions. Participants in the interlaboratory study of method B also received positive and negative control solutions. Each sample was provided as randomly labeled, blind duplicates, and each contained a test portion. Participants were also provided with a method protocol and an electronic evaluation and reporting sheet (MS Excel format). The five test samples were analyzed once under the conditions described in the provided method protocol. Duplicates of samples were analyzed on a different day.

Participants were requested to follow the method protocol exactly. However, the L-ORAC method allows some procedural parameters to be selected within certain limits, such as the direction of fluorescence detection, and the use of an automatic dispenser on the microplate reader. Information regarding the plate readers used by the participants is given in Table S1 (Supporting Information).

\section{Statistical analyses of interlaboratory study data}

Data were rejected from laboratories that did not follow the protocol, as were data whose variance for the Trolox standard solution or negative or positive control solution values (a)

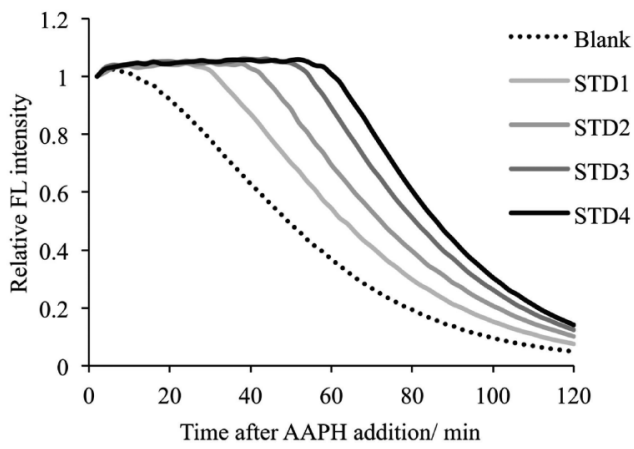

(b)

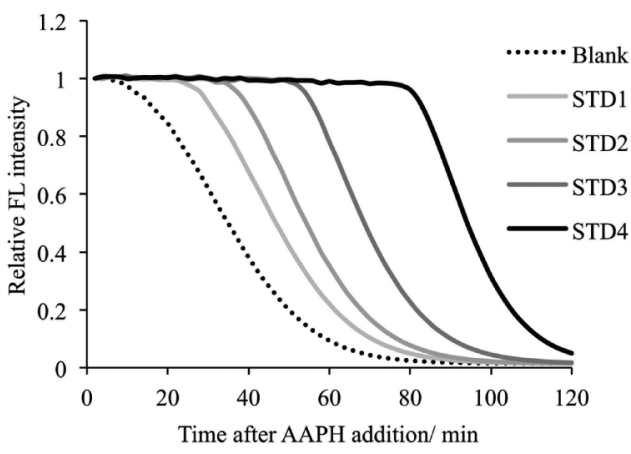

Fig. 2 Fluorescence decay curves of blank and Trolox standard solutions from an interlaboratory test participant for measuring L-ORAC according to method A (a) and method B (b).

were outside the limits. All other data were subjected to the statistical tests described in the internationally harmonized protocol $^{14}$ recommended by CAC/GL 28. The Cochran test was used to identify outlying variances, and the single and double Grubbs tests were used to detect outlying data-set averages. The one-tail test at a probability value of $2.5 \%$ was applied to the Cochran test, and the one-tailed test at a probability value of $1.25 \%$ was applied to the single and double Grubbs tests. Outlier removal was stopped before more than 2/9 laboratories were removed.

\section{Results and Discussion}

Interlaboratory study of L-ORAC measurement according to method A

Although method A showed satisfactory performance in the single-laboratory validation, ${ }^{12}$ several problems were found in the interlaboratory study. The single-laboratory validation of method A showed satisfactory repeatability (repeatability relative standard deviation $\left(\mathrm{RSD}_{\mathrm{r}}\right)$ ranging from 1.3 to $8.0 \%$ ) and intermediate precision (intermediate relative standard deviation $\left(\mathrm{RSD}_{\text {int }}\right.$ ) ranging from 2.1 to $9.5 \%$ ). However, the interlaboratory study demonstrated poor performance for method A ( $\mathrm{RSD}_{\text {int }}$ ranging from 8.4 to $47.3 \%$; reproducibility relative standard deviation $\left(\mathrm{RSD}_{\mathrm{R}}\right)$ ranging from 14.8 to $37.8 \%$ ). The participants identified several serious problems during the measurements. Three of the 16 participating laboratories reported that fluorescence decay was slow, and that the relative fluorescence intensity did not return to zero at $120 \mathrm{~min}$, especially when the concentration of the antioxidant was high (for example, the higher concentration of Trolox standard, 
Table 2 Interlaboratory study results for determinig the antioxidant capacity ( $\mu$ mol Trolox equivalent/L) of test samples by the L-ORAC method B

\begin{tabular}{|c|c|c|c|c|c|c|c|c|c|c|c|c|c|c|c|}
\hline & \multicolumn{15}{|c|}{ Laboratory $^{\mathrm{a}}$} \\
\hline & 1 & 2 & 3 & 4 & 5 & 6 & 7 & 8 & 9 & 10 & 11 & 12 & 13 & 14 & 15 \\
\hline \multirow[t]{2}{*}{$\alpha$-Tocopherol } & 2716 & 2657 & 2719 & 4375 & $6169^{d}$ & 3544 & 2616 & 2731 & 3343 & 3124 & 2594 & 1904 & 3584 & 3566 & 2277 \\
\hline & 2634 & 1897 & 2484 & 3812 & $4955^{\mathrm{d}}$ & 3645 & 2723 & 2713 & 2949 & 2961 & 2596 & 3005 & 3736 & 5239 & 2814 \\
\hline \multirow[t]{2}{*}{$\gamma$-Oryzanol } & 6210 & $5847^{b}$ & 9848 & $10691^{\mathrm{d}}$ & 9556 & 6846 & 5082 & 6279 & 7229 & 8693 & 6737 & 4205 & 7784 & 0 & 4012 \\
\hline & 5852 & $2515^{\mathrm{b}}$ & 6133 & $13780^{\mathrm{d}}$ & 7129 & 6776 & 5553 & 6391 & 7547 & 6646 & 6751 & 8751 & 7379 & 9228 & 5437 \\
\hline \multirow[t]{2}{*}{ Cinnamon } & 3318 & 2815 & 2284 & $3075^{\mathrm{c}}$ & 4312 & 3339 & $2611^{\mathrm{c}}$ & 2837 & 3415 & 3788 & 2854 & 2253 & 3970 & 0 & 1902 \\
\hline & 3126 & 3204 & 2103 & $7929^{c}$ & 4294 & 3392 & $0^{c}$ & 3443 & 2525 & 3431 & 3320 & 3293 & 3726 & 6062 & 2643 \\
\hline \multirow[t]{2}{*}{ Ginger } & 45024 & 35164 & 50732 & $48374^{c}$ & 48475 & 47437 & 45109 & 46295 & 54953 & 40160 & 37528 & 35061 & 57973 & 123018 & 40909 \\
\hline & 43107 & 33378 & 49406 & $69121^{\mathrm{c}}$ & 55775 & 49731 & 35963 & 40804 & 51335 & 42200 & 39816 & 69965 & 54618 & 118218 & 33311 \\
\hline \multirow[t]{2}{*}{ Unpolished rice } & 1011 & 1015 & 721 & $1204^{c}$ & 1488 & 1059 & 907 & 979 & 1097 & 1106 & 1003 & 634 & 1128 & 902 & 859 \\
\hline & 1037 & 1300 & 809 & $1892^{\mathrm{c}}$ & 1337 & 1100 & 914 & 841 & 1125 & 1176 & 1044 & 1189 & 1097 & 843 & 1021 \\
\hline
\end{tabular}

a. Results of laboratories 1 - 11 were used for the statistical analyses. Results of laboratories 12 - 15 were excluded, because positive or negative controls of laboratories 12 and 15, and variations of standard curve of laboratories 13 and 14 were beyond the tolerable ranges. Laboratory 16 failed to measure L-ORAC values of test samples. b. Abnormal values removed prior to statistical analysis. c. Cochran outlier. d. Single Grubbs outlier.

as shown in Fig. 2a). Fluorescence decay should return to the baseline before the end of the measurement to obtain a reproducible net AUC value. In addition, 5 of the 16 participating laboratories reported that repeated measurements with fine adjustment of the dilution ratios were necessary to obtain an approximate L-ORAC value in the first-step measurement. In this step, the test sample solution was arbitrarily diluted into the range of the standard curve. However, the dynamic range of the standard curve covered only a four-fold difference in concentration (Table 1), and thus it was sometimes difficult to find the optimum dilution ratio. To solve these technical issues, method A was modified to establish method B. In addition, the poor performance of method $A$ suggests that the L-ORAC values from failed measurements can be statistically analyzed. Thus, in method B, an L-ORAC measurement was judged to have failed when either the coefficient of variation of the net AUC of the Trolox standard solution or the L-ORAC value of the negative or positive control solution was beyond the tolerable range shown in the Experimental section.

\section{Interlaboratory study of L-ORAC measurement according to} method B

Method B used a lower concentration of fluorescein $(77.5 \mathrm{nmol} / \mathrm{L})$ and a higher concentration of AAPH $(82.4 \mathrm{mmol} / \mathrm{L})$ for radical production compared to method A (Table 1); consequently, the fluorescence intensity decayed faster after the addition of AAPH compared to method A (Fig. 2). In addition, positive and negative controls were used in method $\mathrm{B}$, and the participants were requested to ensure that their L-ORAC values were within the tolerable range (for details, see Experimental).

Individual values obtained at 15 of the 16 participating laboratories are given in Table 2; one laboratory did not report the L-ORAC values due to technical issues. The relative standard deviation of the net AUCs of the Trolox standard solutions were above $15 \%$ at two of the 16 laboratories (laboratories 13 and 14), and the estimated L-ORAC values of the negative or positive control solutions were beyond the tolerable range at two of the 16 laboratories (laboratories 12 and 15). Thus, the results from these laboratories were excluded from the statistical analysis. Because these laboratories used different makes and models of microplate readers (Table S1), these exclusions are likely not to be due to defects in a specific type of reader.

Since one result for $\gamma$-oryzanol from laboratory 2 in Table 2 was double the value of the replicate result, the central laboratory judged these results as being abnormal, and thus excluded them from the statistical analysis. Thus, data sets for $\alpha$-tocopherol solution, and cinnamon, ginger, and unpolished rice extracts from 11 participating laboratories, and for $\gamma$-oryzanol solution from 10 participating laboratories, were subjected to statistical tests described in the internationally harmonized protocol. ${ }^{14}$ The results from two laboratories for cinnamon extract, and one laboratory each for ginger and unpolished rice extract, were removed as Cochran outliers (Table 2). The results for $\alpha$-tocopherol and $\gamma$-oryzanol solutions from one laboratory were removed as single Grubbs outliers (Table 2). Calculations for the intermediate precision and reproducibility, as defined by the protocol, ${ }^{12}$ were performed on the remaining results. $\mathrm{RSD}_{\text {int }}$ and $\mathrm{RSD}_{\mathrm{R}}$ of the test samples ranged from 7.0 to $16.7 \%$ and from 14.8 to $19.4 \%$, respectively (Table 3 ). Both precision parameters were smaller than those of method A.

The precision data obtained in the interlaboratory study were compared with the predicted levels of precision obtained from the Horwitz equation. Because the L-ORAC value is the relative antioxidant capacity of a sample relative to that of Trolox, 1 gram of antioxidant was occasionally equivalent to more than 1 gram of Trolox. In method B, the L-ORAC value was determined based on the converged dilution ratio, which shows the same net AUC as $50 \mu \mathrm{mol} / \mathrm{L}$ Trolox by plotting the dilution ratios against their net AUCs in a double-log diagram. Therefore, the predicted $\mathrm{RSD}_{\mathrm{R}}$ of each sample was calculated from $50 \mu \mathrm{mol} / \mathrm{L}$ of Trolox. The predicted $\mathrm{RSD}_{\mathrm{R}}$ was calculated to be $10.94 \%$ according to the Horwitz equation. The HorRat value, the ratio of $\mathrm{RSD}_{\mathrm{R}}$ to the predicted $\mathrm{RSD}_{\mathrm{R}}$, can be used as a performance parameter, indicating the acceptability of the precision of the method. A HorRat value of $<2$ usually indicates satisfactory reproducibility of the method. The HorRat values of the test samples ranged from 1.35 to 1.78 (Table 3). Since all HorRat values of the test samples were lower than 2, the L-ORAC measurement by method $\mathrm{B}$ was validated by an interlaboratory test in accordance with the internationally harmonized protocol. ${ }^{14}$ 
Table 3 Statistical analysis of data from an interlaboratory study for determinig the antioxidant capacity by the L-ORAC method B

\begin{tabular}{lccccc}
\hline & \multicolumn{5}{c}{ Material } \\
\cline { 2 - 6 } & $\alpha$-Tocopherol & $\gamma$-Oryzanol & Cinnamon & Ginger & Unpolished rice \\
\hline Mean & 2942 & 6959 & 3267 & 44620 & 1053 \\
Numbers of laboratories & $10(1)$ & $9(1)$ & $9(2)$ & $10(1)$ & $10(1)$ \\
$S_{\text {int }}{ }^{\mathrm{a}}$ & 241 & 1163 & 230 & 3190 & 84 \\
$S_{\mathrm{R}}{ }^{\mathrm{c}}$ & 571 & 1279 & 583 & 6605 & 185 \\
$\mathrm{RSD}_{\text {int }}{ }^{\mathrm{d}}$ & 8.2 & 16.7 & 7.0 & 7.2 & 8.0 \\
RSD $_{\mathrm{R}}{ }^{\mathrm{e}}$ & 19.4 & 18.4 & 17.9 & 14.8 & 17.6 \\
HorRat $^{\mathrm{f}}$ & 1.78 & 1.68 & 1.63 & 1.35 & 1.61 \\
\hline
\end{tabular}

Values obtained after removal of outliers identified by Cochran test and single Grubbs test. a. Retained after outlier laboratories removed, and number of outlier laboratories in parentheses. b. $S_{\text {int }}=$ Intermediate precision standard deviation, $\mu$ mol Trolox equivalent/L. c. $S_{\mathrm{R}}=$ Reproducibility standard deviation, $\mu \mathrm{mol}$ Trolox equivalent $/ \mathrm{L}$. d. $\mathrm{RSD}_{\mathrm{int}}=$ Intermediate precision relative standard deviation, $\%$. e. $\mathrm{RSD}_{\mathrm{R}}=$ Reproducibility relative standard deviation, \%. f. HorRat $=\mathrm{RSD}_{\mathrm{R}} /$ predicted $\mathrm{RSD}_{\mathrm{R}}$. Predicted $\mathrm{RSD}_{\mathrm{R}}$ was calculated from the highest concentrations of Trolox standard solution $(50 \mu \mathrm{mol} / \mathrm{L})$. The mass fraction of $50 \mu \mathrm{mol} / \mathrm{L}$ Trolox was calculated by multiplying volume concentration by molecular weight of Trolox, and predicted $\mathrm{RSD}_{\mathrm{R}}$ was calculated to be $10.94 \%$ using the Horwitz equation.

\section{Acknowledgements}

This work was supported by a grant-in-aid "A Scheme to Revitalize Agriculture and Fisheries in Disaster Area through Deploying Highly Advanced Technology" from the Ministry of Agriculture, Forestry and Fisheries of Japan, and by JSPS KAKENHI Grant Number 26282200. We express our appreciation to the following collaborators for their participation in the study: Yuko Kino (Kagome Co.), Yuji Takahashi (Japan Food Research Laboratories), Shinji Owaki (Food Analysis Technology Center SUNATEC), Keiichi Negoro (Industrial Technology Center of Wakayama Prefecture), Ryo Nakagawa (Taiyo Kagaku Co.), Masao Sasaki (Thermo Scientific Co.), Toshihiko Shoji (National Institute of Fruit Tree Science, National Agriculture and Food Research Organization), Satoshi Shimizu (National Agricultural Research Center for Tohoku Region, National Agriculture and Food Research Organization), Yoichi Nokata (Western Region Agricultural Research Center, National Agriculture and Food Research Organization), Koji Ueda (Institute of Vegetable and Tea Sciences, National Agriculture and Food Research Organization), Rie Kurata (Kyushu Okinawa Agricultural Research Center, National Agriculture and Food Research Organization), Koji Ishiguro (Hokkaido Agricultural Research Center, National Agriculture and Food Research Organization), Yumiko Konno (National Food Research Institute, National Agriculture and Food Research Organization), and Noriyuki Furutani (Kyoto Prefectural Agriculture, Forestry and Fisheries Technology Center). We also express appreciation to Dr. Yasuhiro Suzuki (NARO Institute of Crop Science) for providing rice samples.

\section{Supporting Information}

Variation in the plate reader parameters between laboratories.
These materials are available free of charge on the Web at http:// www.jsac.or.jp/analsci/.

\section{References}

1. D. D. Rio, C. Agnoli, and N. Pellegrini, J. Nutr., 2011, 141, 118.

2. Y. Wang, M. Yang, S. G. Lee, C. G. Davis, S. I. Koo, M. L. Fernandez, J. S. Volek, and O. K. Chun, Eur. J. Nutr., 2014, 53, 1363.

3. B. Ou, M. Hampsch-Woodill, and R. L. Prior, J. Agric. Food Chem., 2001, 49, 4619.

4. D. Huang, B. Ou, M. Hampsch-Woodill, J. A. Flanagan, and R. L. Prior, J. Agric. Food Chem., 2002, 50, 4437.

5. B. Halliwell, Annu. Rev. Nutr., 1996, 16, 33.

6. D. Huang and B. Ou, J. Agric. Food Chem., 2002, 50, 1815.

7. P. I. Pfitzner, P. I. Francz, and H. K. Biesalski, Biochim. Biophys. Acta, 2000, 1474, 163.

8. X. Wu and G. Beecher, J. Agric. Food Chem., 2004, 52, 4026.

9. G. W. Burton and M. G. Traber, Annu. Rev. Nutr., 1990, 10, 357.

10. M. V. Eberhardt, K. Kobira, A. S. Keck, J. A. Juvik, and E. H. Jeffery, J. Agric. Food Chem., 2005, 53, 7421.

11. J. Watanabe, T. Oki, J. Takebayashi, K. Yamasaki, Y. Takano-Ishikawa, A. Hino, and A. Yasui, Anal. Sci., 2012, 28, 159.

12. J. Watanabe, T. Oki, J. Takebayashi, K. Yamasaki, Y. Takano-Ishikawa, A. Hino, and A. Yasui, Biosci. Biotechnol. Biochem., 2013, 77, 857.

13. D. Jimenez-Alvarez, F. Giuffrida, F. Vanrobaeys, P. Golay, C. Cotting, A. Lardeau, and B. Keely, J. Agric. Food Chem., 2008, 56, 3470.

14. W. Horwirz, Pure Appl. Chem., 1995, 67, 331. 